\title{
Diabetes mellitus and incidence of kidney cancer: a meta-analysis of cohort studies
}

\author{
S. C. Larsson • A. Wolk
}

Received: 26 October 2010 / Accepted: 20 December 2010 /Published online: 28 January 2011

(C) Springer-Verlag 2011

\begin{abstract}
Aims/hypothesis Diabetes is associated with increased risk of cancer at several sites, but its association with cancer of the kidney is unclear. We performed a systematic review with meta-analysis to examine the association between diabetes and incidence of kidney cancer.

Methods Pertinent studies were identified by searching PubMed (from January 1966 to December 2010) and reviewing the reference lists of relevant articles. We included cohort studies reporting RR estimates and 95\% CI (or data to calculate them) of the association between diabetes and kidney cancer incidence. Summary RRs were calculated using a random-effects model.

Results Nine cohort studies met the inclusion criteria. Analysis of all studies showed that compared with individuals without diabetes, patients with diabetes had a statistically significant increased risk of kidney cancer (RR $1.42,95 \%$ CI 1.06-1.91). There was heterogeneity among studies $(p<0.001$ for heterogeneity). The association was stronger in women (RR 1.70, 95\% CI 1.47-1.97) than in men (RR 1.26, 95\% CI 1.06-1.49). When restricting the analysis to studies that had adjusted for body mass index $(n=3)$ or cigarette smoking $(n=3)$, the RRs were 1.12 (95\% CI 0.99 1.27 ) and 1.29 (95\% CI 1.05-1.58), respectively.

Conclusions/interpretation This meta-analysis indicates a positive association between diabetes and risk of kidney cancer. Future research should attempt to establish whether this association is causal.
\end{abstract}

S. C. Larsson $(\bowtie) \cdot$ A. Wolk

Division of Nutritional Epidemiology,

The National Institute of Environmental Medicine,

Karolinska Institutet,

Box 210, SE-171 77 Stockholm, Sweden

e-mail: susanna.larsson@ki.se
Keywords Cohort studies · Diabetes mellitus . Epidemiology $\cdot$ Kidney cancer $\cdot$ Kidney neoplasms . Meta-analysis $\cdot$ Renal cell carcinoma $\cdot$ Review

\section{Introduction}

The incidence of kidney cancer has been increasing worldwide over the past few decades [1]. Besides obesity, cigarette smoking and hypertension, which are associated with greater risk of kidney cancer [2-5], the causes of this disease are largely unknown.

Like kidney cancer, type 2 diabetes is a serious and growing health problem worldwide. Obesity and cigarette smoking are also positively associated with risk of type 2 diabetes [6, 7]. Evidence from epidemiological studies indicates that diabetic patients have an increased risk of cancer at several sites, including cancers of the colon and rectum [8], pancreas [9], liver [10], bladder [11], breast [12] and endometrium [13]. Whether diabetes is also a risk factor for kidney cancer remains unclear. We used metaanalysis to systematically review published cohort studies examining the association between diabetes and incidence of kidney cancer.

\section{Methods}

Retrieval of studies We conducted a computerised search of the PubMed database for studies published in any language between January 1966 and December 2010 using the search term 'diabetes', combined with 'kidney cancer', 'renal cell cancer' or 'renal cell carcinoma'. We also manually reviewed the references of relevant publications to search for further studies and reviewed publications on diabetes 
and risk of total and specific cancers to search for results on kidney cancer incidence.

Inclusion and exclusion criteria In this meta-analysis, we included studies that fulfilled the following criteria: (1) publication presented original data from a cohort study; (2) the exposure of interest was diabetes mellitus; (3) the outcome of interest was incidence of kidney or renal cell cancer; and (4) RR estimates with CIs or enough data to calculate them were reported. We summarised results from cohort studies because these are less prone to bias than case-control studies. We did not consider studies in which the exposure of interest was mainly or solely type 1 diabetes, defined as diagnosis before 30 years of age. Studies on mortality rates from kidney or renal cell cancer were not included. In the event of multiple publications from the same study population, the most recent publication with the largest number of kidney cancer cases was included in the meta-analysis.

Data extraction We extracted the following information from each publication: the first author's last name, publication year, study location, age and sex of participants, sample size (cases and cohort size), diabetes assessment and type of diabetes, variables controlled for in the analysis and RR estimates with CIs for the association between diabetes and kidney cancer incidence. From each study, we extracted the RR estimate that was adjusted for the greatest number of potential confounders.

Statistical analysis Summary RRs and their 95\% CIs were calculated with the method of DerSimonian and Laird using the assumptions of a random-effects model that considers within-study and between-study variation [14]. We investigated possible heterogeneity in results across studies by using the $Q$ and $I^{2}$ statistics [15]. $I^{2}$ is the proportion of total variation contributed by between-study variation [15]. To assess for publication bias, we applied regression methods to determine funnel plot asymmetry as proposed by Egger et al. [16]. A $p$ value of less than 0.10 was considered statistically significant. All statistical analyses were performed with STATA version 10.1 (StataCorp, College Station, TX, USA).

\section{Results}

We identified 15 potentially relevant cohort studies [17-31]. Six studies were excluded because of overlapping publications from the same study population $[17,19]$ or because the outcome was cancer mortality [28-31]. Thus, a total of nine cohort studies met our inclusion criteria (Table 1). Four studies were conducted in Europe, three in Asia and two in North America. The study population in six studies consisted of both sexes [18, 20, 23-25, 27], two studies included men only $[22,26]$ and one study included women only [20]. The outcome was renal cell carcinoma in three studies [20, 23, 25], and total or unspecified kidney cancer in six studies [18, 21, 22, 24, 26, 27]. Two studies used a 1 year latency period in all analyses $[18,26]$.

Analysis of all studies showed that, compared with individuals without diabetes, those with diabetes had a statistically significant increased risk of kidney cancer (1.42, $95 \%$ CI 1.06-1.91; Fig. 1). There was statistically significant heterogeneity among studies ( $p<0.001$ for heterogeneity; $I^{2}=$ $95.2 \%)$. In analysis stratified by sex, a significantly stronger $(p=0.01)$ positive association was observed in women (RR $1.70,95 \%$ CI $1.47-1.97 ; p=0.98$ for heterogeneity; $I^{2}=0 \%$ ) than in men (RR 1.26, 95\% CI 1.06-1.49; $p=0.01$ for heterogeneity; $I^{2}=69.9 \%$ ). The association between diabetes and kidney cancer was significantly stronger $(p=0.05)$ in studies with a standardised incidence ratio as the measure of relative risk (RR 1.70, 95\% CI 1.21-2.40; $p<0.001$ for heterogeneity; $I^{2}=94.3 \%$ ) than in studies with a rate ratio as the measure of relative risk (RR 1.17, 95\% CI 1.00-1.37; $p=$ 0.25 for heterogeneity; $\left.I^{2}=23.1 \%\right)$. In stratified analysis by outcome, the RRs were $1.12(95 \%$ CI $0.57-2.22 ; p=0.18$ for heterogeneity; $\left.I^{2}=37.1 \%\right)$ in studies of renal cell carcinoma and 1.50 (95\% CI 1.09-2.08; $p<0.001$ for heterogeneity; $I^{2}=$ $96.9 \%$ ) in studies of total or unspecified kidney cancer. The difference in risk estimates by outcome was not statistically significant $(p=0.45)$. We found no evidence of publication bias (Egger's test: $p=0.75$ ).

Obesity and cigarette smoking are risk factors for diabetes and for kidney cancer, and thus potential confounders of the relationship between diabetes and risk of kidney cancer. When we restricted the analysis to the three studies that had adjusted for body mass index or obesity $[23,24,26]$, the RR was 1.12 ( $95 \%$ CI $0.99-1.27 ; p=0.39$ for heterogeneity; $I^{2}=2.4 \%$ ). Among the three studies that controlled for cigarette smoking [22-24], the pooled RR was 1.29 (95\% CI $1.05-1.58 ; p=0.66$ for heterogeneity; $I^{2}=0 \%$ ).

Six studies examined the risk of kidney cancer by interval between start of follow-up and kidney cancer diagnosis [18, 23-27]. When the RR estimates obtained after excluding 1, 2 or 5 years of follow-up were combined $[18,23-27]$, the pooled RR of kidney cancer was 1.48 (95\% CI 1.13-1.94; $p<0.001$ for heterogeneity; $I^{2}=91.5 \%$ ). Three studies examined the effect of excluding the first 5 years of follow-up [24] or used a latency period of 5 years between diabetes diagnosis or hospitalisation and diagnosis of kidney cancer [26, 27]. When the risk estimates from those studies were combined, the pooled RR of kidney cancer associated with a long history of diabetes ( $\geq 5$ years) was 1.35 (95\% CI $0.98-1.86 ; p=0.005$ for heterogeneity; $I^{2}=76.5 \%$ ). 


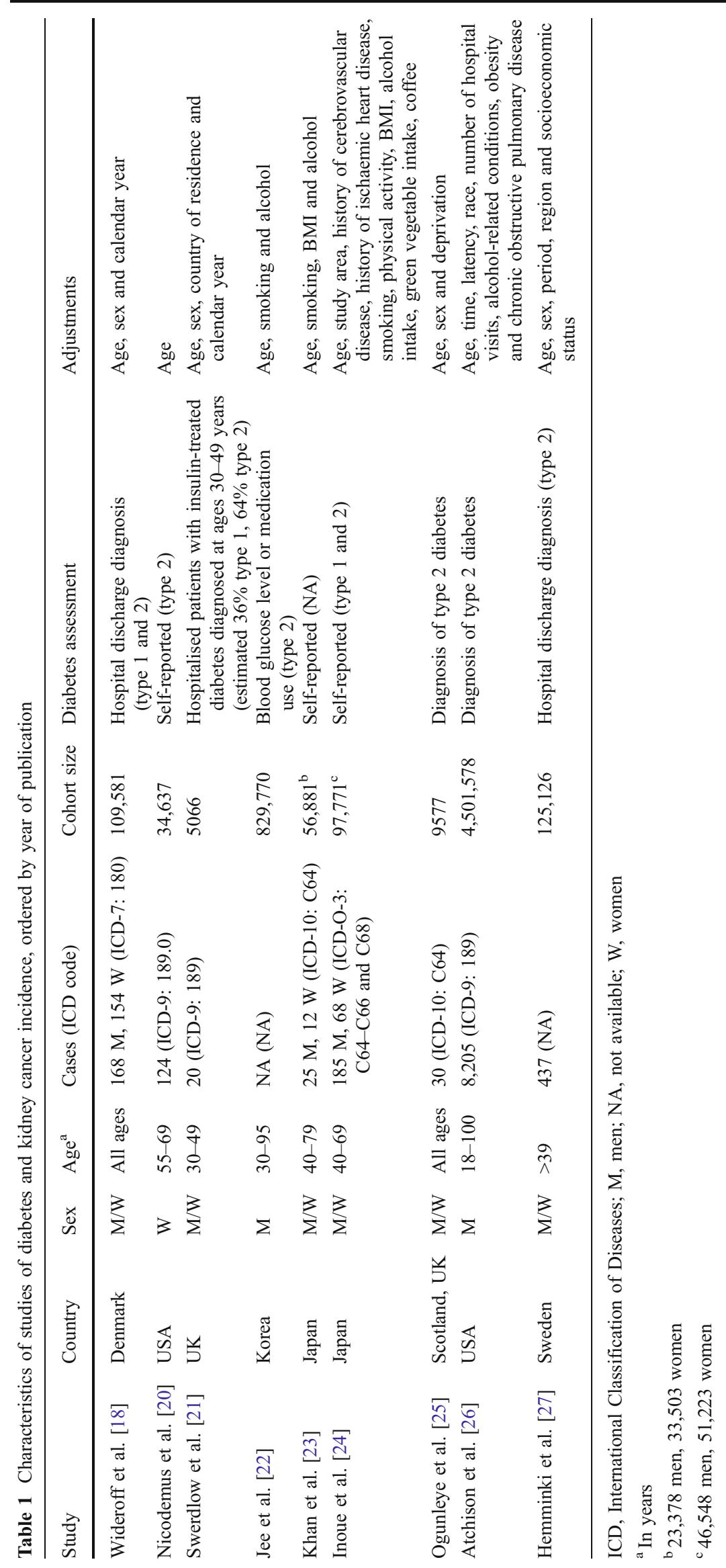


Fig. 1 RRs for the association between diabetes mellitus and kidney cancer incidence in cohort studies. The black squares and horizontal lines represent the study-specific relative risk and corresponding 95\% CI, respectively. The size of the black squares reflects the weight of the respective study, measured by the inverse of the variance. The diamond represents the pooled relative risk with its $95 \%$ CI. Test for heterogeneity among studies: $Q=229.85 ; p<0.001 ; I^{2}=95.2 \%$. $\mathrm{M}$, men; W, women

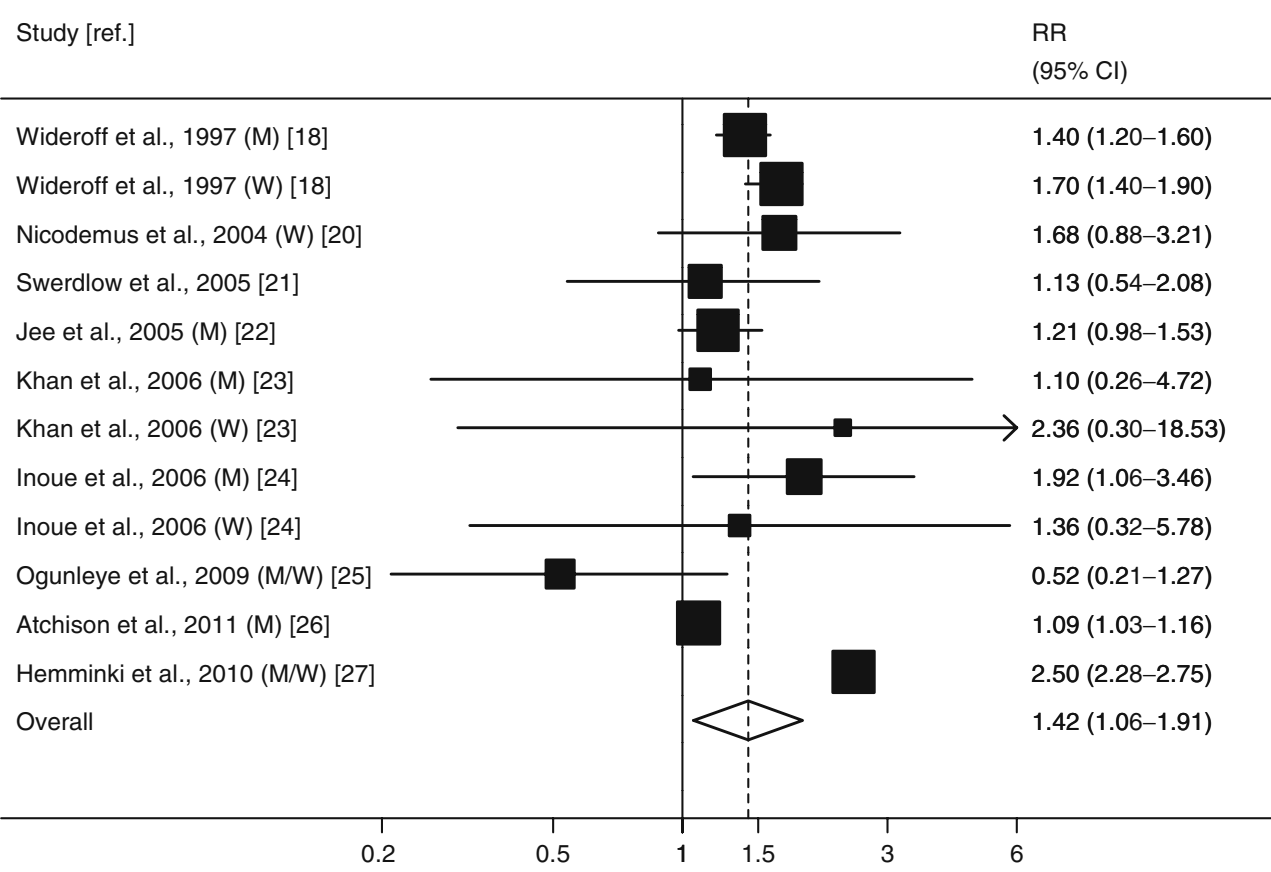

\section{Discussion}

The findings of this meta-analysis of nine cohort studies indicate that diabetes is associated with a $42 \%$ increased risk of kidney cancer. The positive association was stronger in women than in men. It was also stronger in studies with a standardised incidence ratio as the measure of relative risk than in studies with a rate ratio, possibly due to lack of adjustment for potential confounders such as obesity and cigarette smoking.

Whether the association between diabetes and kidney cancer is causal is unclear. In this respect, several points should be considered. First, because our analyses were based on observational studies, confounding cannot be excluded as a potential explanation for the observed positive association between diabetes and risk of kidney cancer. Obesity has consistently been associated with increased risk of diabetes [7] and kidney cancer [4]. Only three studies controlled for body mass index or obesity [23, $24,26]$. The relation between diabetes and kidney cancer was weaker and not statistically significant when we restricted the analysis to those studies, suggesting that the observed association may in part be due to confounding from obesity. Cigarette smoking is another shared risk factor between type 2 diabetes and kidney cancer [2, 6]. When risk estimates from the three studies that adjusted for smoking were combined [22-24], the association between diabetes and kidney cancer was weaker (RR 1.29) than the overall result including all studies (RR 1.42).

Second, detection bias may also have contributed to the observed association between diabetes and kidney cancer risk because diabetic patients are under increased medical surveillance. If medical surveillance bias is present, kidney cancer would tend to be diagnosed at an earlier stage in diabetic patients than in those without diabetes. Two studies examined the risk of kidney cancer by interval between diabetes diagnosis [26] or last hospitalisation for type 2 diabetes [27] and diagnosis of kidney cancer. In the study by Atchison et al. [26], the risk of kidney cancer declined over time and was no longer significantly increased at 10 years after diagnosis of diabetes. The RRs of kidney cancer for an interval between diagnoses of diabetes and kidney cancer were 1.24 (95\% CI 1.11-1.38) after 2 to 5 years, 1.09 (95\% CI 0.97-1.22) after 6 to 10 years and 1.00 (95\% CI 0.91-1.10) after $>10$ years [26]. In the study by Hemminki et al. [27], the standardised incidence ratio of kidney cancer declined from 2.50 with no latency period to 1.44 for a 5 year latency period. In the study by Ogunleye et al. [25], the RR of kidney cancer comparing diabetes with no diabetes was $0.52(95 \%$ CI $0.21-1.27)$ when including all cases and 0.25 (95\% CI 0.06-1.06) when excluding cases diagnosed within the first year of followup. In contrast, two other studies found a stronger positive association between diabetes and kidney cancer risk after excluding the first 2 or 5 years of follow-up [23, 24]. Because of these inconsistent results, it remains unclear whether the observed positive association between diabetes and kidney cancer risk is due to detection bias.

Because diabetes is an underdiagnosed disease, some misclassification of exposure is likely, which would tend to attenuate any true association between diabetes and kidney cancer. Diabetes was diagnosed and assessed differently in the studies. In some studies, diabetes was self-reported [20, 23, 24], whereas other studies assessed diabetes through 
diabetes hospitalisation or diagnosis [18, 21, 25-27]. Some studies included type 1 and type 2 diabetes, which would lead to an attenuation of the risk estimates if only type 2 diabetes is a risk factor for kidney cancer. In fact, a population-based cohort study in Sweden found no excess risk of kidney cancer in hospitalised type 1 diabetes patients [32]. Moreover, because total kidney cancer was assessed in most studies, the overall association would be attenuated if diabetes were a risk factor for renal cell (parenchyma) or renal pelvis cancer only. In this meta-analysis, diabetes was more strongly associated with total and unspecified kidney cancer than renal cell cancer. Only one study reported results for renal cell and renal pelvis cancer [19]. In that study, the association between diabetes and risk of kidney cancer was similar for cancers of the parenchyma (standardised incidence ratio $1.5 ; 95 \%$ CI $1.3-1.7$ ) and pelvis (standardised incidence ratio 1.4; 95\% CI 1.0-1.9) [19].

There was heterogeneity among study results. The heterogeneity of risk estimates may be due to different adjustment for potential confounders and different mixtures of type 1 and type 2 diabetic participants. Furthermore, some studies included both sexes, whereas others included only men or only women. If the association is stronger in women, as suggested in this meta-analysis, different proportions of men and women in the studies may in part account for the observed heterogeneity.

Inherent in any meta-analysis of published data is the possibility of publication bias, that is small studies with null results tend not to be published. However, we found no evidence of publication bias in this meta-analysis.

Diabetes may affect the risk of kidney cancer by increasing insulin resistance and blood levels of insulin and IGF-I [33, 34]. Experimental evidence indicates that insulin could affect malignant transformation or tumour growth through its anabolic and anti-apoptotic growth factor effects or by increasing the levels of bioactive IGFI $[35,36]$. IGF-I may stimulate cell proliferation and inhibit apoptosis [37]. IGF-I levels have been positively associated with risk of several cancers (e.g. breast and prostate cancer) $[38,39]$. However, a study among older middle-aged male smokers found that low serum IGF-I levels were associated with increased risk of kidney cancer [40]. This finding suggests that IGF-I may not explain the positive association between diabetes and kidney cancer risk. Diabetes may also potentially increase the risk of kidney cancer through hypertension, which has been positively associated with risk of kidney cancer [3,5]. Indeed, type 2 diabetes and hypertension frequently coexist [41].

In summary, the available epidemiological evidence indicates that individuals with diabetes have an increased risk of developing kidney cancer. The possibility that the association may be due to confounding or detection bias cannot be ruled out. Additional large cohort studies that adjust for obesity and other potential confounders as well as assessing latency between diabetes and kidney cancer are needed to establish a potential causal relationship between diabetes and kidney cancer risk. Future research should also assess whether the association differs for renal cell and renal pelvis cancer.

Acknowledgements This work was supported by research grants from the Swedish Cancer Foundation.

Duality of interest The authors declare that there is no duality of interest associated with this manuscript.

\section{References}

1. Mathew A, Devesa SS, Fraumeni JF Jr, Chow WH (2002) Global increases in kidney cancer incidence, 1973-1992. Eur J Cancer Prev 11:171-178

2. Hunt JD, van der Hel OL, McMillan GP, Boffetta P, Brennan P (2005) Renal cell carcinoma in relation to cigarette smoking: meta-analysis of 24 studies. Int J Cancer 114:101-108

3. Chow WH, Gridley G, Fraumeni JF Jr, Jarvholm B (2000) Obesity, hypertension, and the risk of kidney cancer in men. N Engl J Med 343:1305-1311

4. Bergström A, Hsieh CC, Lindblad P, Lu CM, Cook NR, Wolk A (2001) Obesity and renal cell cancer-a quantitative review. Br J Cancer 85:984-990

5. Corrao G, Scotti L, Bagnardi V, Sega R (2007) Hypertension, antihypertensive therapy and renal-cell cancer: a meta-analysis. Curr Drug Saf 2:125-133

6. Willi C, Bodenmann P, Ghali WA, Faris PD, Cornuz J (2007) Active smoking and the risk of type 2 diabetes: a systematic review and meta-analysis. JAMA 298:2654-2664

7. Abdullah A, Peeters A, de Courten M, Stoelwinder J (2010) The magnitude of association between overweight and obesity and the risk of diabetes: a meta-analysis of prospective cohort studies. Diabetes Res Clin Pract 89:309-319

8. Larsson SC, Orsini N, Wolk A (2005) Diabetes mellitus and risk of colorectal cancer: a meta-analysis. J Natl Cancer Inst 97:1679-1687

9. Huxley R, Ansary-Moghaddam A, Berrington de Gonzalez A, Barzi F, Woodward M (2005) Type-II diabetes and pancreatic cancer: a meta-analysis of 36 studies. Br J Cancer 92:2076-2083

10. El-Serag HB, Hampel H, Javadi F (2006) The association between diabetes and hepatocellular carcinoma: a systematic review of epidemiologic evidence. Clin Gastroenterol Hepatol 4:369-380

11. Larsson SC, Orsini N, Brismar K, Wolk A (2006) Diabetes mellitus and risk of bladder cancer: a meta-analysis. Diabetologia 49:2819-2823

12. Larsson SC, Mantzoros CS, Wolk A (2007) Diabetes mellitus and risk of breast cancer: a meta-analysis. Int J Cancer 121:856-862

13. Friberg E, Orsini N, Mantzoros CS, Wolk A (2007) Diabetes mellitus and risk of endometrial cancer: a meta-analysis. Diabetologia 50:1365-1374

14. DerSimonian R, Laird N (1986) Meta-analysis in clinical trials. Control Clin Trials 7:177-188

15. Higgins JP, Thompson SG (2002) Quantifying heterogeneity in a meta-analysis. Stat Med 21:1539-1558

16. Egger M, Davey Smith G, Schneider M, Minder C (1997) Bias in meta-analysis detected by a simple, graphical test. BMJ 315:629-634

17. Adami HO, McLaughlin J, Ekbom A et al (1991) Cancer risk in patients with diabetes mellitus. Cancer Causes Control 2:307-314 
18. Wideroff L, Gridley G, Mellemkjaer L et al (1997) Cancer incidence in a population-based cohort of patients hospitalized with diabetes mellitus in Denmark. J Natl Cancer Inst 89:1360-1365

19. Lindblad P, Chow WH, Chan J et al (1999) The role of diabetes mellitus in the aetiology of renal cell cancer. Diabetologia 42:107-112

20. Nicodemus KK, Sweeney C, Folsom AR (2004) Evaluation of dietary, medical and lifestyle risk factors for incident kidney cancer in postmenopausal women. Int J Cancer 108:115-121

21. Swerdlow AJ, Laing SP, Qiao Z et al (2005) Cancer incidence and mortality in patients with insulin-treated diabetes: a UK cohort study. Br J Cancer 92:2070-2075

22. Jee SH, Ohrr H, Sull JW, Yun JE, Ji M, Samet JM (2005) Fasting serum glucose level and cancer risk in Korean men and women. JAMA 293:194-202

23. Khan M, Mori M, Fujino Y et al (2006) Site-specific cancer risk due to diabetes mellitus history: evidence from the Japan Collaborative Cohort (JACC) Study. Asian Pac J Cancer Prev 7:253-259

24. Inoue M, Iwasaki M, Otani T, Sasazuki S, Noda M, Tsugane S (2006) Diabetes mellitus and the risk of cancer: results from a large-scale population-based cohort study in Japan. Arch Intern Med 166:1871-1877

25. Ogunleye AA, Ogston SA, Morris AD, Evans JM (2009) A cohort study of the risk of cancer associated with type 2 diabetes. Br J Cancer 101:1199-1201

26. Atchison EA, Gridley G, Carreon JD, Leitzmann MF, McGlynn KA (2011) Risk of cancer in a large cohort of U.S. veterans with diabetes. Int J Cancer 128:635-643

27. Hemminki K, Li X, Sundquist J, Sundquist K (2010) Risk of cancer following hospitalization for type 2 diabetes. Oncologist 15:548-555

28. Kessler II (1970) Cancer mortality among diabetics. J Natl Cancer Inst 44:673-686

29. Verlato G, Zoppini G, Bonora E, Muggeo M (2003) Mortality from site-specific malignancies in type 2 diabetic patients from Verona. Diabetes Care 26:1047-1051
30. Coughlin SS, Calle EE, Teras LR, Petrelli J, Thun MJ (2004) Diabetes mellitus as a predictor of cancer mortality in a large cohort of US adults. Am J Epidemiol 159:1160-1167

31. Lam EK, Batty GD, Huxley RR et al. (2011) Associations of diabetes mellitus with site-specific cancer mortality in the AsiaPacific region. Ann Oncol. doi:10.1093/annonc/mdq405

32. Shu X, Ji J, Li X, Sundquist J, Sundquist K, Hemminki K (2010) Cancer risk among patients hospitalized for type 1 diabetes mellitus: a population-based cohort study in Sweden. Diabet Med 27:791-797

33. Macaulay VM (1992) Insulin-like growth factors and cancer. Br J Cancer 65:311-320

34. Bach LA, Rechler MM (1992) Insulin-like growth factors and diabetes. Diabetes Metab Rev 8:229-257

35. Pollak M (2008) Insulin and insulin-like growth factor signalling in neoplasia. Nat Rev Cancer 8:915-928

36. Calle EE, Kaaks R (2004) Overweight, obesity and cancer: epidemiological evidence and proposed mechanisms. Nat Rev Cancer 4:579-591

37. Giovannucci E (2001) Insulin, insulin-like growth factors and colon cancer: a review of the evidence. J Nutr 131:3109S-3120S

38. Key TJ, Appleby PN, Reeves GK, Roddam AW (2010) Insulinlike growth factor 1 (IGF1), IGF binding protein 3 (IGFBP3), and breast cancer risk: pooled individual data analysis of 17 prospective studies. Lancet Oncol 11:530-542

39. Rowlands MA, Gunnell D, Harris R, Vatten LJ, Holly JM, Martin RM (2009) Circulating insulin-like growth factor peptides and prostate cancer risk: a systematic review and meta-analysis. Int $\mathrm{J}$ Cancer 124:2416-2429

40. Major JM, Pollak MN, Snyder K, Virtamo J, Albanes D (2010) Insulin-like growth factors and risk of kidney cancer in men. $\mathrm{Br} \mathrm{J}$ Cancer 103:132-135

41. Epstein M, Sowers JR (1992) Diabetes mellitus and hypertension. Hypertension 19:403-418 